\title{
Beam energy scan with asymmetric collision at RHIC
}

\author{
C. Liu, J. Alessi, E. Beebe, M. Blaskiewicz, J. M. Brennan, K. A. Brown, \\ D. Bruno, J. Butler, R. Connolly, T. D’Ottavio, K. A. Drees, W. Fischer,
} C. J. Gardner, D. M. Gassner, X. Gu, Y. Hao, M. Harvey, T. Hayes, H. Huang, R. Hulsart, P. Ingrassia, J. Jamilkowski, J. S. Laster, V. Litvinenko, Y. Luo, M. Mapes, G. J. Marr, A. Marusic, G. McIntyre, K. Mernick, R. Michnoff, M. Minty, C. Montag, J. Morris, C. Naylor, S. Nemesure, I. Pinayev, V. Ranjbar, D. Raparia, G. Robert-Demolaize, T. Roser, P. W. Sampson, J. Sandberg, V. Schoefer, F. Severino, T. Shrey, K. Smith, S. Tepikian, Y. Than, P. Thieberger, J. Tuozzolo, G. Wang, Q. Wu, A. Zaltsman, K. Zeno, S. Zhang, W. Zhang

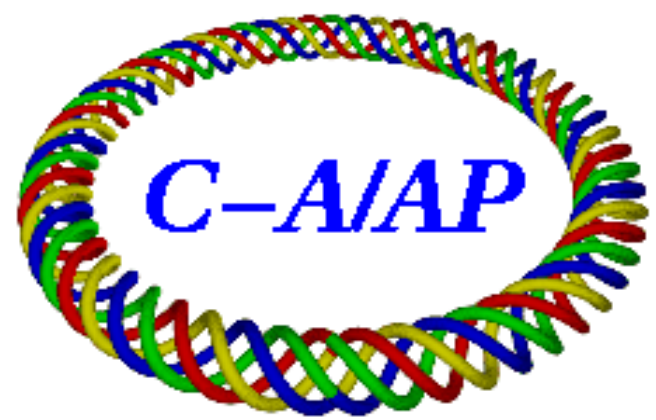

\section{Collider-Accelerator Department Brookhaven National Laboratory Upton, NY 11973}

\section{U.S. Department of Energy Office of Science, Office of Nuclear Physics}

Notice: This document has been authorized by employees of Brookhaven Science Associates, LLC under Contract No. DE-SC0012704 with the U.S. Department of Energy. The United States Government retains a nonexclusive, paid-up, irrevocable, world-wide license to publish or reproduce the published form of this document, or allow others to do so, for United States Government purposes. 


\section{BEAM ENERGY SCAN WITH ASYMMETRIC COLLISION AT RHIC}

C. Liu, J. Alessi, E. Beebe, M. Blaskiewicz, J. M. Brennan, K. A. Brown, D. Bruno, J. Butler, R. Connolly, T. D’Ottavio, K. A. Drees, W. Fischer, C. J. Gardner, D. M. Gassner, X. Gu, Y. Hao, M. Harvey, T. Hayes, H. Huang, R. Hulsart, P. Ingrassia, J. Jamilkowski, J. S. Laster, V. Litvinenko, Y. Luo, M. Mapes, G. J. Marr, A. Marusic, G. McIntyre, K. Mernick, R. Michnoff, M. Minty, C. Montag, J. Morris, C. Naylor, S. Nemesure, I. Pinayev, V. Ranjbar, D. Raparia, G. Robert-Demolaize, T. Roser, P. W. Sampson, J. Sandberg, V. Schoefer, F. Severino, T. Shrey, K. Smith, S. Tepikian, Y. Than, P. Thieberger, J. Tuozzolo, G. Wang, Q. Wu, A. Zaltsman, K. Zeno, S. Zhang, W. Zhang, Brookhaven National Lab, Upton, NY, USA.

\section{Abstract}

A beam energy scan of deuteron-gold collision, with center-of-mass energy at 19.6, 39, 62.4 and $200.7 \mathrm{GeV} / \mathrm{n}$, was performed at the Relativistic Heavy Ion Collider in 2016 to study the threshold for quark-gluon plasma (QGP) production. The lattice, RF, stochastic cooling and other subsystems were in different configurations for the various energies. The operational challenges changed with every new energy. The operational experience at each energy, the operation performance, highlights and lessons of the beam energy scan are reviewed in this report.

\section{INTRODUCTION}

The 6 weeks deuteron-gold beam energy scan was in-between two periods of the $200 \mathrm{GeV} / \mathrm{n}$ gold-gold collision [1], respectively 10 weeks and 1 week. The center-of-mass energies of the collision are 19.6, 39, 62.4 and 200.7 $\mathrm{GeV} / \mathrm{n}$ for the scan. The first two energies are below while the latter two are above transition energy $\left(\gamma_{t} \sim 23.5\right)$. Since the chance of pre-fire [2] drops with beam energy, it was planned to scan energy in ascending order (19.6, 39, 62.4, $200.7 \mathrm{GeV} / \mathrm{n}$ ) for better protection of the detectors. However, the order was changed as requested by both experiments based on the physics priority. The running time of each energy was about 1 week. The $200.7 \mathrm{GeV} / \mathrm{n}$ program started on May $9^{\text {th }}$ and ended on $20^{\text {th }}$. The $62.4 \mathrm{GeV} / \mathrm{n}$ program started on May $20^{\text {th }}$ and ended on $27^{\text {th }}$. The 19.6 GeV/n program started on May $27^{\text {th }}$ and ended on June $8^{\text {th }}$. The $39 \mathrm{GeV} / \mathrm{n}$ program started on Jun $8^{\text {th }}$ and ended on June $17^{\text {th }}$.

The luminosity goals for the energy scan are based on the projection [3], which is based on past runs and scaling laws. The luminosity goals and projected beam parameters are shown in Table 1.

Table 1: Projected and achieved beam parameters and luminosities for the beam energy scan with deuteron-gold collision.

\begin{tabular}{|c|c|c|c|c|c|c|c|c|}
\hline \multirow{2}{*}{$\begin{array}{c}\mathrm{E} \\
(\mathrm{GeV} / \mathrm{n})\end{array}$} & \multicolumn{3}{|c|}{ Projected beam parameters and luminosities } & \multicolumn{3}{|c|}{ Achieved beam parameters and luminosities } \\
\cline { 2 - 9 } & $\mathrm{N}\left(10^{9}\right)$ & $\beta^{*}(\mathrm{~m})$ & L_week & SC status & $\mathrm{N}\left(10^{9}\right)$ & $\beta^{*}(\mathrm{~m})$ & L_week & SC status \\
\hline 19.6 & $140 * 0.9$ & 3 & $1.5 \mathrm{nb}^{-1}$ & off & $190 * 2.1$ & 3.5 & $2.8 \mathrm{nb}^{-1}$ & off \\
\hline 39 & $140 * 1.5$ & 1.5 & $5.0 \mathrm{nb}^{-1}$ & 3D cooling & $160^{*} 2.1$ & 2 & $9.4 \mathrm{nb}^{-1}$ & YV cooling, No re- \\
bucketing for d
\end{tabular}




\section{OPERATION REVIEW}

The deuteron beam was provided by Tandem while the gold beam was provided by the Electron Beam Ion Source (EBIS). Tandem provided gold beam in the period of two EBIS cathode problems ( $\sim 2$ days each). The deuteron beam went through 4-2 bunch merge in AGS; the gold beam went through 12-6-2 merge in AGS. The operation of the Booster and AGS in 2016 was reviewed in Ref. [4, 5]. The beam parameters for deuteron and gold beams in Booster, AGS and RHIC were summarized in Ref. [6].

\section{Orbit and aperture}

The aperture restriction came from the facts that the beam orbits are tilted horizontally and the reduced aperture for the CeC PoP undulator [7]. The top view of the orbits of the deuteron (blue) and gold beam (yellow) at IR2 is shown in Fig. 1. The common angle of the beams with respect to the straight line from the center of DX magnet on one side to the other is $1 \mathrm{mrad}$. The beams are separated vertically by $8 \mathrm{~mm}$ at IRs except for IR6 and 8 .

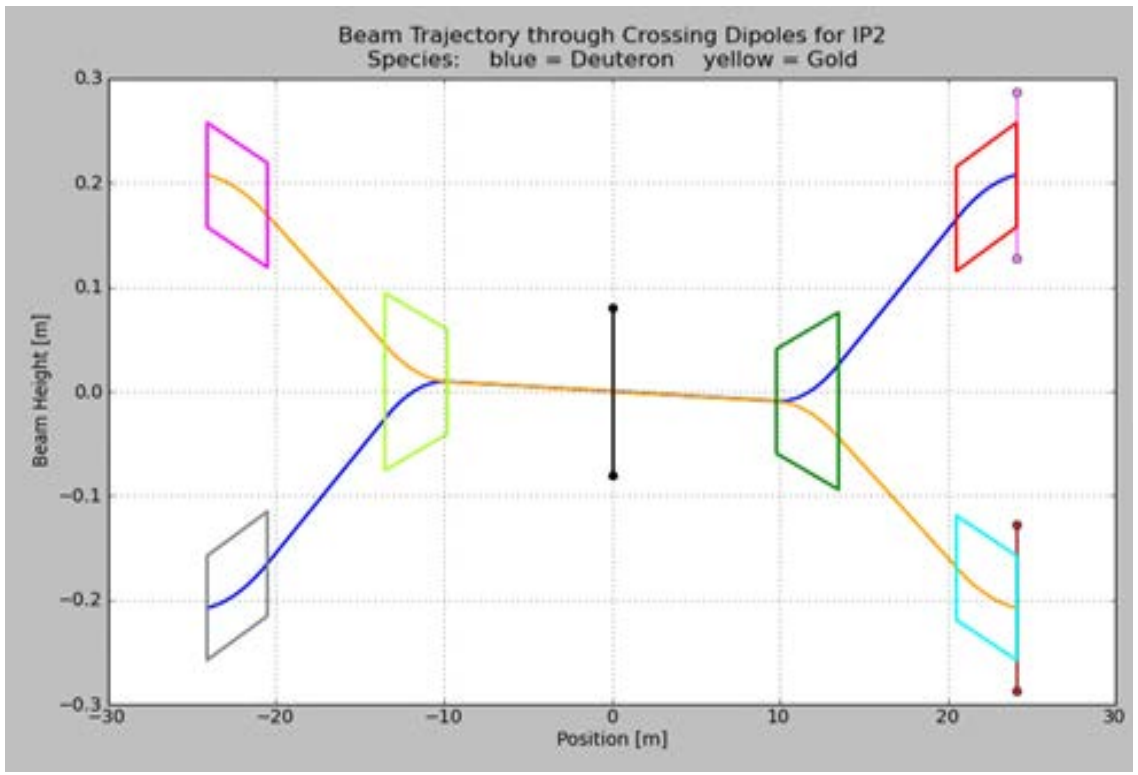

Figure 1: The orbits of the deuteron and gold beam at IR2. The magnets $\sim 10 \mathrm{~m}$ away from IP are DX magnets; 20 $\mathrm{m}$ away from IP are D0 magnets.

The layout of the CeC PoP experiment is shown in Fig. 2. The aperture of the undulator beam pipe (-4.2 to $4.2 \mathrm{~m}$ around IP2) is in diamond shape with inner sides of $28 \mathrm{~mm}$. Beam study with gold beam revealed the aperture restriction was worse than expected. Following that, an optical imaging was set up to inspect the inside of the vacuum chamber and found loose RF fingers. Also the survey found that the chamber was elevated by $\sim 2.5 \mathrm{~mm}$ between the $2^{\text {nd }}$ and $3^{\text {rd }}$ undulators. After re-alignment of the chamber, the RF fingers retracted and there was no obstacle visible inside. However, beam orbit scan still indicated smaller aperture at the lower part of the chamber.
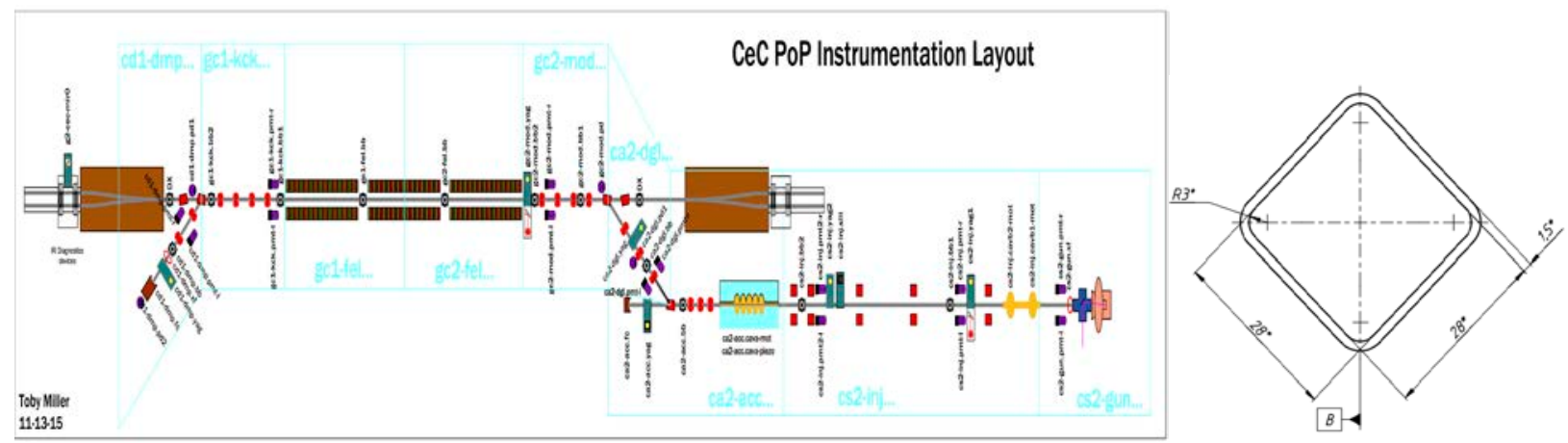

Figure 2: The layout of the CeC PoP experiment and the aperture of the undulator beam pipe. 
Several measures were taken to combat this aperture restriction. We reduced the beam common angle from $1 \mathrm{mrad}$ to $0.7 \mathrm{mrad}$ by setting up an angle bump at IR2 for both beams. With thorough aperture studies with beam, a new injection scheme was conceived and applied operationally: We Inject the IBS-robust deuteron beam first with no vertical offset. Once blue ring is filled with deuteron, we move beam down to $-3 \mathrm{~mm}$ vertical offset at IR2. Then inject gold beam in yellow ring with $+5 \mathrm{~mm}$ vertical offset. Tape sequence was programmed to go through these steps automatically.

\section{Beam intensity}

The merging scheme for gold beam in AGS was upgraded from 8-4-2 to 12-6-2 for more intensity per bunch [4, 5]. The new scheme was briefly tested in 2015. With better controlling of the longitudinal emittance, it was operational for RHIC gold-gold and deuteron-gold program in 2016. With bunch intensity of 3.0e9 from AGS, the bottleneck of the gold beam intensity is the transition crossing in RHIC. The bunch intensity was limited to be 2.3e9 due to the Landau cavity power amplifier. The characteristic of the Landau voltage is shown in Fig. 3 when the intensity limit is approached.

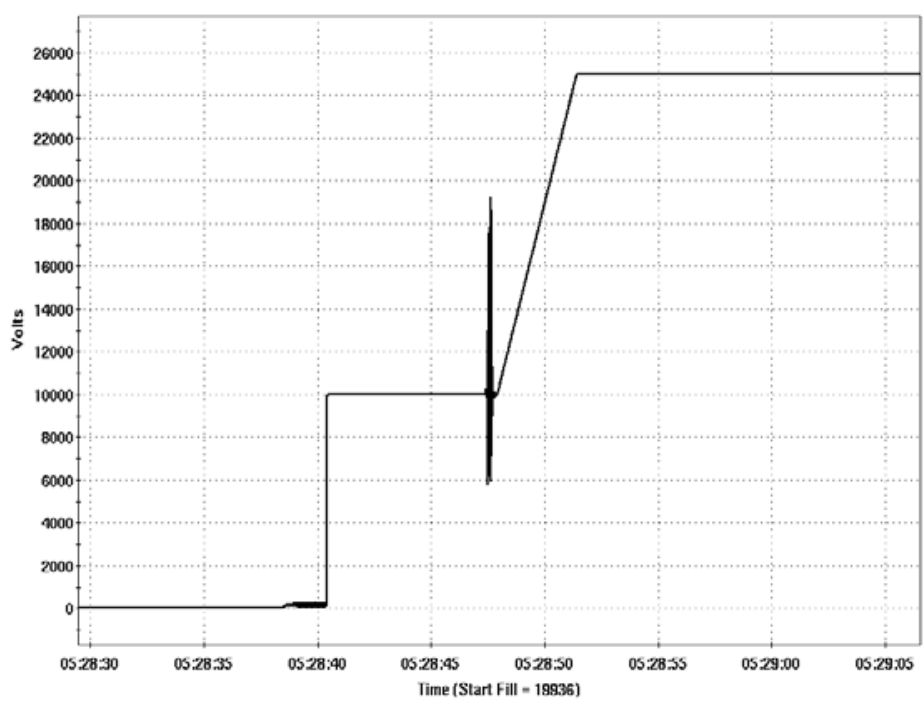

Figure 3: The voltage of the Landau cavity during beam acceleration; the spikes around 05:28:47 are at the time of transition crossing.

The deuteron bunch intensity can reach 2.0E11 with only one bunch merge in AGS. The deuteron intensity was limited at transition crossing in RHIC as well. The deuteron beam is accelerated to top energy with the revolution frequency locked with that of the gold beam [8]. Therefore, it crosses transition energy at a time slightly different from the gold beam due to path length difference of them. The equivalent bunch intensity limit for deuteron beam is 1.8E11 assuming the same longitudinal profile. The observed intensity limit at transition for deuteron is lower than expected at the start of the energy scan program. It was found this was due to the short bunch length of the deuteron beam. The deuteron beam went through a 4-2 merge in AGS [5], therefore the longitudinal emittance is smaller than that of gold beam and the bunch is shorter. To better match the 3D sizes of the two beams in RHIC and for more deuteron intensity, the deuteron bunch was lengthened to match the gold beam. The longitudinal profiles measured in AGS are shown in Fig. 4. As a result, the intensity limit due to Landau cavity at transition crossing reached $1.8 \mathrm{E} 11$ as expected. 

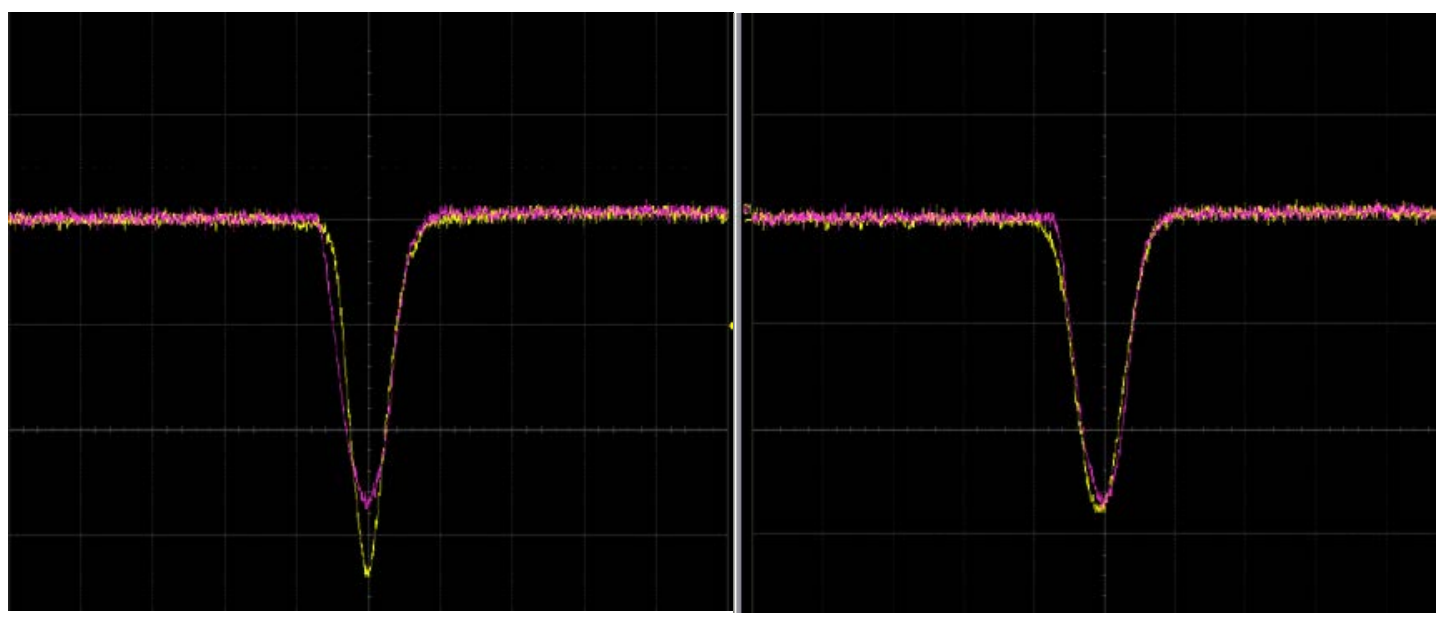

Figure 4: The longitudinal profile of deuteron (yellow) and gold (purple) bunch measured in AGS, on the left are the original ones before lengthening, on the right are the ones with deuteron bunch lengthened.

\section{Beam emittance}

The 3D beam emittance evolution was dominated by intra-beam scattering [9] for the gold beam, not for deuteron beam. The counter-measure for intra-beam scattering is the 3D stochastic cooling [10], which are operational in both rings. However stochastic cooling doesn't work for the deuteron beam due to the high bunch intensity. The configuration of stochastic cooling in yellow ring for gold beam is summarized in Table 1 . The longitudinal and horizontal cooling was on for the $200.7 \mathrm{GeV} / \mathrm{n}$ program. The longitudinal and vertical cooling was on for the 62.4 $\mathrm{GeV} / \mathrm{n}$ program. Excessive beam loss at the stochastic cooling kicker prevented engaging the horizontal cooling at $62.4 \mathrm{GeV} / \mathrm{n}$. Global coupling was introduced in the machine for cooling of emittance in both planes [11]. Only the vertical cooling was on for $39 \mathrm{GeV} / \mathrm{n}$. Longitudinal IBS growth was slowed down due to equilibrium below transition (Fig. 12). No cooling was engaged at $19.6 \mathrm{GeV} / \mathrm{n}$ due to too large beam size. The transverse emittance evolutions are shown in following for all the energies. The decrease of the vertical emittance at $19.6 \mathrm{GeV} / \mathrm{n}$ is believed to be the result of beam scraping in vertical plane by aperture limits (like collimator, $\mathrm{CeC}$ undulator aperture at IR2).

Figure 5: Beam transverse emittance evolution of the two beams for the $200.7 \mathrm{GeV} / \mathrm{n}$ program. 


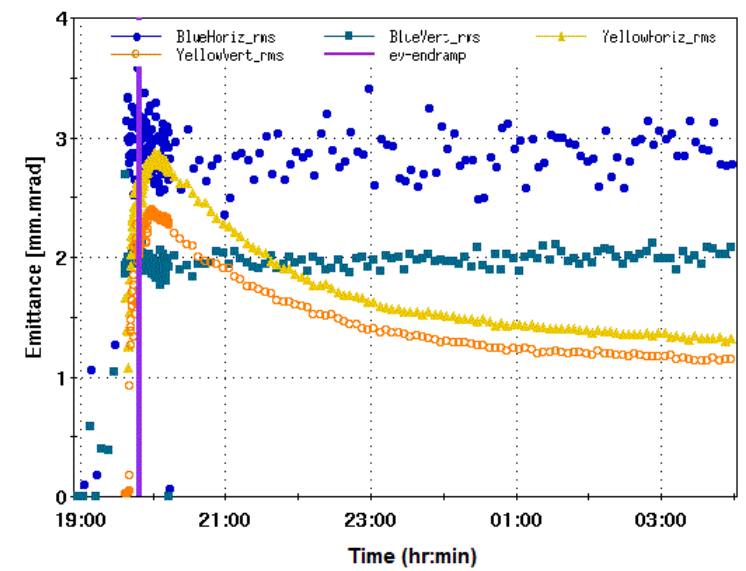

Figure 6: Beam transverse emittance evolution of the two beams for the $62.4 \mathrm{GeV} / \mathrm{n}$ program.

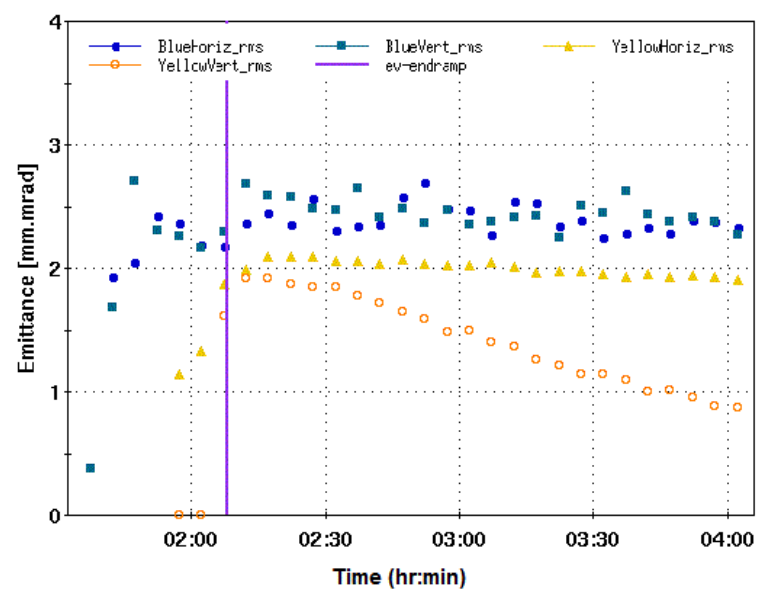

Figure 7: Beam transverse emittance evolution of the two beams for the $19.6 \mathrm{GeV} / \mathrm{n}$ program.

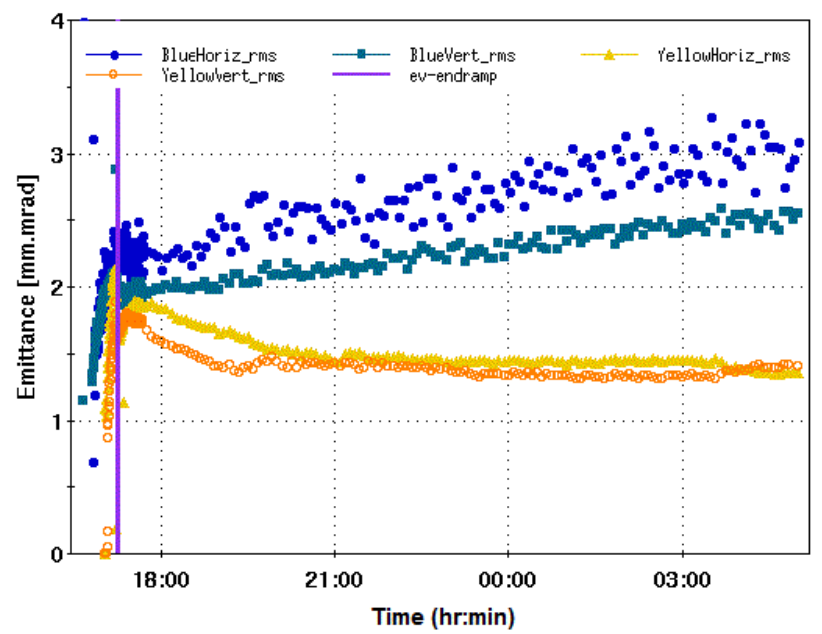

Figure 8: Beam transverse emittance evolution of the two beams for the $39 \mathrm{GeV} / \mathrm{n}$ program. 


\section{The $56 \mathrm{~Hz}$ cavity}

The voltage of the beam driven $56 \mathrm{MHz}$ cavity [12] was limited by the HOM damper. The cavity was operated without the HOM damper in 2016. With significant effort of beam study during gold-gold program, the cavity was running operationally starting from the deuteron-gold energy scan program. The extra longitudinal focusing provided by the cavity reduces the bunch length, and slows down the intra-beam scattering rate. There was an instant luminosity gain of $11 \%$ due to $56 \mathrm{MHz}$ cavity. The instant gain of the PHENIX $10 \mathrm{~cm}$ vertex is around $15 \%$ due to $56 \mathrm{MHz}$ cavity.

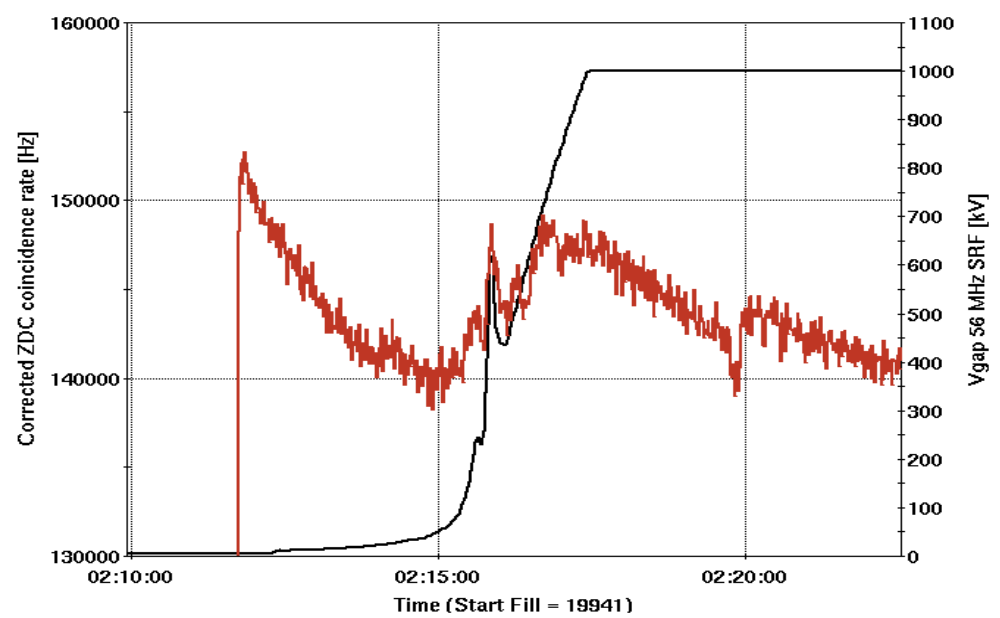

Figure 9: The improvement of collision rate for PHENIX with the $56 \mathrm{MHz}$ cavity engaged.

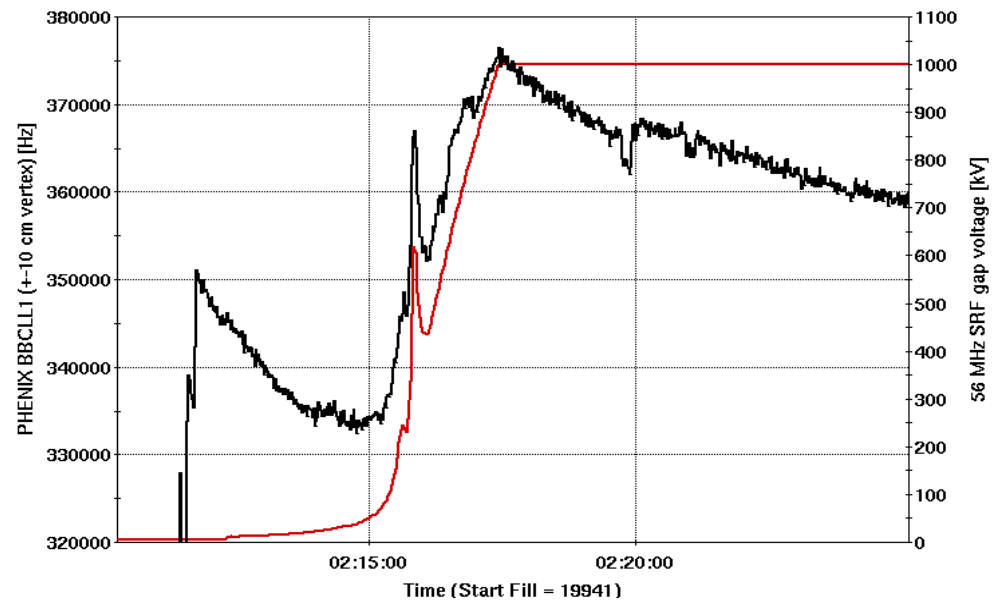

Figure 9: The improvement of collision rate in the $+/-10 \mathrm{~cm}$ vertex range for PHENIX with the $56 \mathrm{MHz}$ cavity engaged.

\section{Actions for protecting detectors}

There were some actions taken for asymmetric collision to protect detectors from pre-fire of abort kicker [13]. Prefire protection orbit bumps were employed in both rings for $200.7 \mathrm{GeV} / \mathrm{n} \mathrm{d}$-Au operations. The newly designed PHENIX MPC protection circuit raised protection threshold by a factor of 400. The Sector-8 DX magnet was moved by $9 \mathrm{~mm}$ so that the aperture for Au beam in d-Au configuration is the same as that in Gold-gold configuration. The lattice was redesigned to adjust phase advances between abort kickers and masks [14] ( 73 degree compared with 180 degree in 2015) so that the masks are more effective in case of pre-fire. The dipole magnet quench protection diode failure happened during gold-gold program is believed to be related to the off-momentum beam loss at the protection bump. Therefore, a mechanical serial switch for the abort kicker is planned to protect the detectors by eliminating the chance of pre-fire at top energy. 


\section{Notes for $200.7 \mathrm{GeV} / \mathrm{n}$}

The yellow injection kicker ceramic pipe was replaced during the setup period of the $200.7 \mathrm{GeV} / \mathrm{n}$ deuteron-gold program. It was no longer limiting the intensity of the gold beam any more due to high vacuum pressure and resulting beam loss from beam-gas interactions. The IR2 aperture limit was overcome with all the actions taken (see details in subsection "orbit and aperture") and the beam loss on undulator was under control. The $56 \mathrm{MHz}$ cavity has been operational since the first $200.7 \mathrm{GeV} / \mathrm{n}$ deuteron-gold physics store, with voltage raised to $1 \mathrm{MV}$ in the last three stores.

\section{Notes for $62.4 \mathrm{GeV} / \mathrm{n}$}

It was possible to deliver long stores with very good luminosity lifetime with stochastic cooling engaged. Turn-on time of SC cooling was crucial because the early beam loss was very large without cooling. The excessive early beam loss prevented the engagement of the cooling. To be able to turn the cooling on right after collision, we delayed the ramping of the storage cavity voltage [15] and also fine-tuned collimators settings [16] to reduce the early beam loss. Beam instability at injection was suppressed by large chromaticity.

\section{Notes for $19.6 \mathrm{GeV} / \mathrm{n}$}

The store length is $~ 1.5 \mathrm{hrs}$, compared to $0.5 \mathrm{hr}$ Gold-gold store in 2011. The injection efficiency and beam lifetime were improved with the new lattice with $3.5 \mathrm{~m}$ beta star [17]. The lifetime of deuteron is better than that of $\mathrm{Au}$. Initially, beams were injected into collision, however losing too much blue beam while injecting yellow beam. We switched to fill with separation bumps and then put beams into collision. The disadvantage was that one loses beam sometimes mostly due to coherence when establishing beam collision. It is recommended not to run magnets through hysteresis regularly for low energy programs, however, we need to do it when magnets dropped to zero due to various reasons. The machine at low energy lacks long term stability. It is hard to reproduce a store with the same good settings. The response amplitude of beam transfer function (BTF) measurement at $19.6 \mathrm{GeV} / \mathrm{n}$ was much lower than that at higher energies, which is a proof the tune spread is wider. It turned out to be the biggest contributor to the beam lifetime. Pushing chromaticity close to zero and engaging octupoles reduced the loss rates (Fig. 11). And the spectrum from BTF was narrower which indicates narrower tune spread (Fig. 12). However, we ran into instability problem when ramping into these configurations. It was recommended to apply this for low energy programs; the remedy for instability is transverse damper [15].

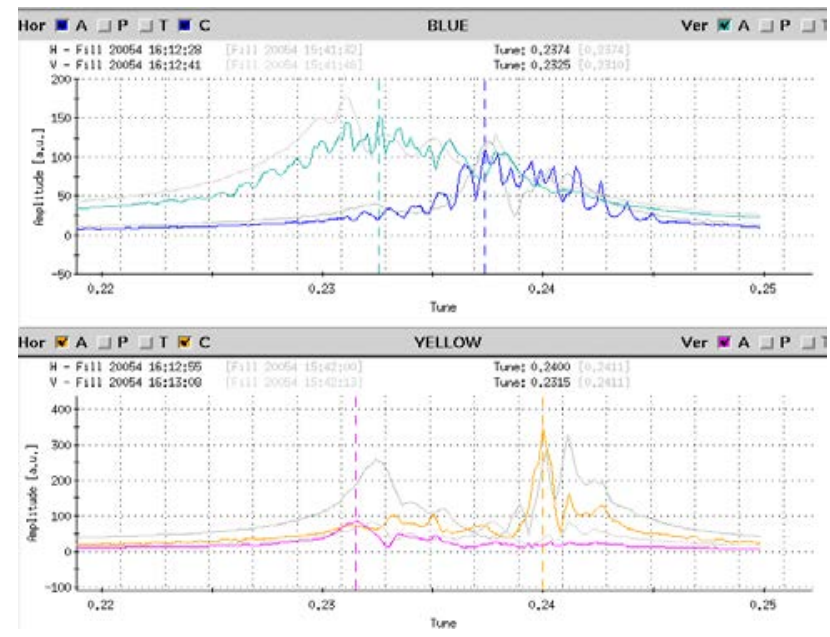

Figure 10: Beam transfer function with relatively large (grey) and small (yellow) chromaticities in yellow horizontal plane. 


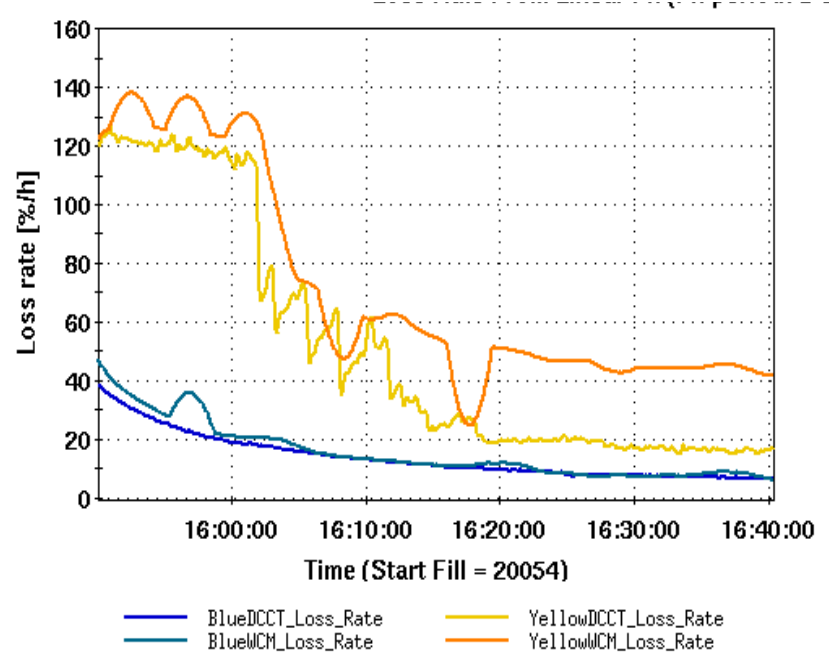

Figure 11: The reduction of beam loss rate by pushing yellow horizontal chromaticity close to zero and engaging octupole magnets.

\section{Notes for $39 \mathrm{GeV} / \mathrm{n}$}

This is the lowest energy that SC cooling was engaged in RHIC. There are two challenges: beam energy is too close to transition energy, beam sizes are large at kickers. A new lattice [18] was designed to fulfil the requirements of large slip factor and small beam sizes at kickers. With vertical cooling only, the horizontal emittance got cooled due to coupling and the longitudinal IBS growth rate is reduced (Fig. 12). The gold beam re-bucketing works fine, the deuteron beam re-bucketing cannot be performed due to high beam loss. The beam was not re-bucketed for the deuteron beam since bunch is relatively short at the energy which is close transition [19]. PHENIX narrow vertex ratio is almost identical with or without blue beam re-bucketing.

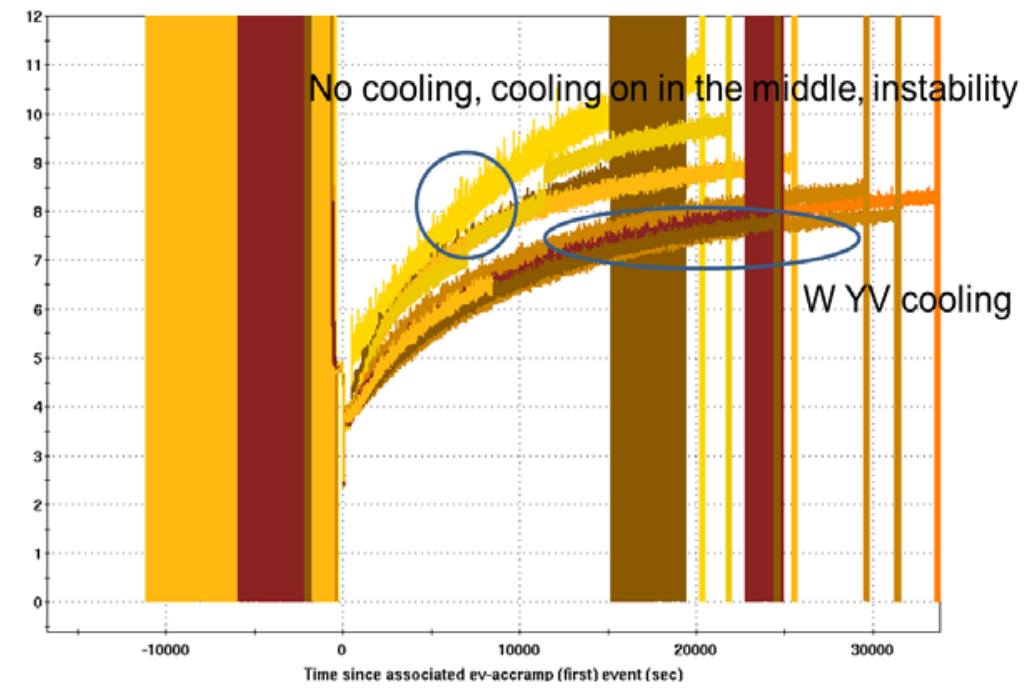

Figure 12: The bunch length evolution without and with vertical cooling engaged for gold beam; the longitudinal IBS growth is slower with vertical cooling.

\section{OPERATION PERFORMANCE}

The switch-overs between different energies were very efficient during deuteron-gold program, even though some happened just before the weekends. The high-efficiency saved us some time so that we switched back to gold-gold operation for a week in the end of the run. The delivered luminosity all exceeded the goals as shown in Table 1 . The luminosity per week of $200.7 \mathrm{GeV} / \mathrm{n}$ was over nine times of that in 2008 deuteron-gold program. 


\section{REFERENCE}

[1] Gu, X., et al., RHIC gold-gold operation at $100 \mathrm{GeV}$ in Run16. NAPAC 2016, Chicago, USA. , 2016.

[2] Zhang, W., et al., Analysis of RHIC beam dump pre-fires. NAPAC2011, New York, USA. , 2016.

[3] W. Fischer et al. RHIC collider projections (FY2016-FY2023). http://www.rhichome.bnl. gov/RHIC/Runs/RhicProjections.pdf.

[4] K. Zeno. Run 16 Tandem gold performance in the injectors and possible improvement with AGS type 6-3-1 bunch merge in the booster. Technical report, C-A/AP/576, 2016.

[5] K. Zeno. Overview and analysis of the 2016 gold run in the booster and AGS. Technical report, C-A/AP/571, 2016.

[6] C. J. Gardner. FY2016 parameters for deuterons and gold ions in booster, AGS, and RHIC. Technical report, CA/AP/575, 2016.

[7] I. Pinayev et al. Commissioning of CeC PoP accelerator. NAPAC, Chicago, USA. 2016.

[8] C. Liu et al., RHIC operation with asymmetric collisions in 2015. In 7th International Particle Accelerator Conference (IPAC’16), Busan, Korea, May 8-13, 2016, pages 1527- 1529.

[9] A. V. Fedotov et al., Experimental studies of IBS in RHIC and comparison with theories. In Proc. of HB2006 Workshop (Tsukuba, Japan, 2006), page 259.

[10] M. Blaskiewicz, J. M. Brennan, and K. Mernick. Three dimensional stochastic cooling in the Relativistic Heavy Ion Collider. Physical review letters, 105(9):094801, 2010.

[11] W. Fischer and Y. Luo. Private communication.

[12] K. Smith. Private communication.

[13] J. Dunlop. Machine protection task force report. Unpublished.

[14] A. Drees et al., RHIC pre-fire protection masks. Technical report, C-A/AP/533, 2015.

[15] K. Mernick. Private communication.

[16] A. Drees. Private communication.

[17] C. Montag. Private communication.

[18] G. Robert-Demolaize. Private communication.

[19] T. Shrey. Private communication. 